\title{
Erratum: Measurements of Chemical Potentials in Superconducting Tin Whiskers*
}

\author{
R. Tidecks \\ I. Physikalisches Institut der Universität Göttingen, Göttingen, West Germany
}

(Received March 20, 1985)

The third from the last sentence on p. 244 should read: However, quantitatively there is a change in the initial slope with which $V_{\mathrm{CE}}\left(I_{\mathrm{AB}}\right)$ develops from $I_{\mathrm{AB}}=0$ in the range where $I_{\mathrm{AB}}$ can be carried in part 1 as a supercurrent.

*This paper appeared in J. Low Temp. Phys. 58, 233 (1985). 